\title{
Adenosine-induced Asystole during AVM Embolization
}

\author{
A Case Series
}

\author{
V. Hellstern ${ }^{1} \cdot$ P. Bhogal ${ }^{2}$ (D) - M. Aguilar Pérez ${ }^{1} \cdot$ M. Alfter ${ }^{1}$ A. Kemmling ${ }^{1} \cdot$ E. Henkes ${ }^{1}$. O. Ganslandt ${ }^{3}$. \\ H. Henkes ${ }^{1,4}$
}

Received: 17 November 2020 / Accepted: 28 April 2021 / Published online: 14 June 2021

(c) The Author(s) 2021

\begin{abstract}
Background Adenosine induced cardiac standstill has been used intraoperatively for both aneurysm and arteriovenous malformation (AVM) surgery and embolization. We sought to report the results of adenosine induced cardiac standstill as an adjunct to endovascular embolization of brain AVMs.

Material and Methods We retrospectively identified patients in our prospectively maintained database to identify all patients since January 2007 in whom adenosine was used to induce cardiac standstill during the embolization of a brain AVM. We recorded demographic data, clinical presentation, Spetzler Martin grade, rupture status, therapeutic intervention and number of embolization sessions, angiographic and clinical results, clinical and radiological outcomes and follow-up information.

Results We identified 47 patients (22 female, 47\%) with average age 42 \pm 17 years (range 6-77 years) who had undergone AVM embolization procedures using adjunctive circulatory standstill with adenosine. In total there were 4 Spetzler Martin grade $1(9 \%), 9$ grade $2(18 \%), 15$ grade $3(32 \%), 8$ grade $4(18 \%)$, and 11 grade $5(23 \%)$ lesions. Of the AVMs six were ruptured or had previously ruptured. The average number of embolization procedures per patient was $5.7 \pm 7.6$ (range 1-37) with an average of 2.6 2.2 (range 1-14) embolization procedures using adenosine. Overall morbidity was $17 \%$ $(n=8 / 47)$ and mortality $2.1 \%(n=1 / 47)$, with permanent morbidity seen in $10.6 \%(n=5 / 47)$ postembolization. Angiographic follow-up was available for 32 patients with no residual shunt seen in $26(81 \%)$ and residual shunts seen in 6 patients $(19 \%)$. The angiographic follow-up is still pending in 14 patients. At last follow-up $93.5 \%$ of patients were mRS $\leq 2$ $(n=43 / 46)$.

Conclusion Adenosine induced cardiac standstill represents a viable treatment strategy in high flow AVMs or AV shunts that carries a low risk of mortality and permanent neurological deficits.
\end{abstract}

Keywords Adenosine $\cdot$ Asystole $\cdot$ AVM $\cdot$ Embolization $\cdot$ Resection

\footnotetext{
Availability of Data There are no further data available for sharing at the current time.

\section{P. Bhogal}

bhogalweb@aol.com

1 Neuroradiological Clinic, Klinikum Stuttgart, Stuttgart, Germany

2 The Royal London Hospital, Barts Health NHS Trust, Whitechapel Road, London, E1 1BB, UK

3 Neurosurgical Clinic, Klinikum Stuttgart, Stuttgart, Germany

4 Medical Faculty, University Duisburg-Essen, Essen, Germany
}

\section{Introduction}

Adenosine, an endogenous purine nucleoside, suppresses sinus and atrioventricular (AV) node conduction and hence can induce transient AV blockade. At low doses (3-12 mg) it is a commonly used intravenous antiarrhythmic agent; however, at higher doses it can trigger ventricular asystole and resultant hypotension or circulatory standstill.

The initial report of adenosine induced hypotension and asystole in neurosurgery were by Pile-Spellman et al. [1] for the endovascular embolization of a high-flow arteriovenous malformation (AVM). Subsequently, numerous reports have documented the use of this technique in neurosurgery, 
principally for the treatment of aneurysms [2-10] but also for AVMs [1, 11-15].

Here we report our results of adenosine induced ventricular asystole from a series of 44 patients.

\section{Methods}

\section{Patient Population}

We performed a retrospective analysis of our prospectively maintained databases to identify all patients who underwent endovascular embolization, either partial or complete, for an AVM. The data set included patients with unruptured and ruptured AVMs as well as those that were found incidentally or presented with nonhemorrhagic neurological symptoms. We identified all patients who were treated between January 2007 and January 2020.

For each patient we recorded demographic data, clinical presentation, Spetzler Martin grade, rupture status, therapeutic intervention and number of embolization sessions, angiographic and clinical results, clinical and radiological outcomes and follow-up information.

\section{Endovascular Treatment}

Informed consent was obtained before the procedure from all patients or their legal representatives unless in the case of emergency treatment. Patients were explicitly informed that induced asystole is an established but off-label use of adenosine. It was explained that amongst the other procedural risks, severe and even life-threatening arrhythmia and the need for emergency electrical cardioversion may result. Patients were also informed that within the personal experience of the senior author with several hundred adenosine asystoles, no clinically relevant complications had occurred.

The preparation of the patients included a routine 12lead electrocardiograph (ECG) examination and in the case of any known or newly detected cardiac abnormality a complete cardiac work-up. At the beginning of the endovascular procedure defibrillator pads were placed on the patient and a defibrillator was connected ready for use. Via the common femoral vein a $4 \mathrm{~F}$ venous access a diagnostic catheter (Tempo4 vertebral, Cordis, Santa Clara, CA, USA) was introduced under fluoroscopy with the tip of the catheter positioned at the level of the right atrium. Invasive intraarterial blood pressure measurement was used by default.

All treatments were performed with the patient under general anesthesia via the right common femoral artery using a short $6 \mathrm{~F}$ sheath and a $6 \mathrm{~F}$ guide catheter. In patients without or outside the acute phase of intracranial hemorrhage, anticoagulation was achieved with a $5000 \mathrm{IU}$ bolus dose of unfractionated heparin at the start of the procedure and subsequent $1000 \mathrm{IU}$ bolus doses every hour to maintain the activated clotting time between 2-2.5 times the baseline. Standard biplane DSA runs were acquired and served to select target vessels for the embolization procedure. In general:

- a reduction of the volume of the AVM nidus,

- an occlusion of as many feeding arteries as possible,

- a reduction of the arteriovenous shunt volume,

- no compromise of the venous drainage of the AVM,

were the goals of the endovascular procedures.

Standard flow directed microcatheters (Marathon/Mirage, Medtronic; Dublin, Ireland, Magic 1.2, Balt Extrusion, Montmorency, France) were used to catheterize the target vessels. The injection of $0.3-0.5 \mathrm{ml}$ Imeron 300 (Bracco Imaging, Konstanz, Germany) with a $2 \mathrm{ml}$ syringe enabled the angioarchitecture of the arteriovenous connection to be visualized. In the case of many small-caliber vessels (plexiform nidus) or a few medium-caliber vessels (microfistulous nidus), embolization was carried out without induced asystole. In the case of a large-caliber high flow arteriovenous connection (macrofistulous nidus), embolization under induced asystole was considered.

In all cases n-butyl-cyanoacrylate (nBCA, Glubran2, GEM; histoacryl, B. Braun, Melsungen, Germany) was used as the occlusive liquid agent. Iodized oil (Lipiodol ultra fluid, Guerbet GmbH, Sulzbach, Germany) was added in equal parts in order to make the occlusive agent radiopaque and to slightly delay the polymerization for better control. Histoacryl polymerizes faster than Glubran2 and was used if this appeared to be advantageous. Glubran2 has a CE mark for intra-arterial embolization, which is not the case for histoacryl. Histoacryl, however, is identical with Trufill nBCA (Cordis), which is approved by the FDA for the embolization of brain AVMs. The mixture of either Glubran2 or histoacryl with Lipiodol is an off-label use. The $\mathrm{nBCA}$ and Lipiodol were drawn into a $1 \mathrm{ml}$ syringe and manually shaken for at least $2 \mathrm{~min}$.

Asystole was induced by a bolus injection of $36 \mathrm{mg}$ adenosine (Adrekar, Sanofi-Aventis) through a $4 \mathrm{~F}$ venous catheter at the level of the right atrium. This dose is used as standard and is independent from age and body weight. In the vast majority of patients $10-20 \mathrm{~s}$ of asystole is achieved. We do not start an infusion of sodium nitroprusside nor do we give hypotensive agents during the procedure.

Before the injection of adenosine the microcatheter is thoroughly flushed with a $5 \%$ glucose solution. While the anesthesiologist injects the adenosine bolus the interventionist fills the dead space of the microcatheter with $\mathrm{nBCA}$ / Lipiodol. Once the electric cardiac standstill begins the interventionist counts to 8 , and then slowly starts to inject the embolic liquid. Visual control is achieved by high frame rate (e.g., 6/s) DSA or, for radioprotection purposes, un- 


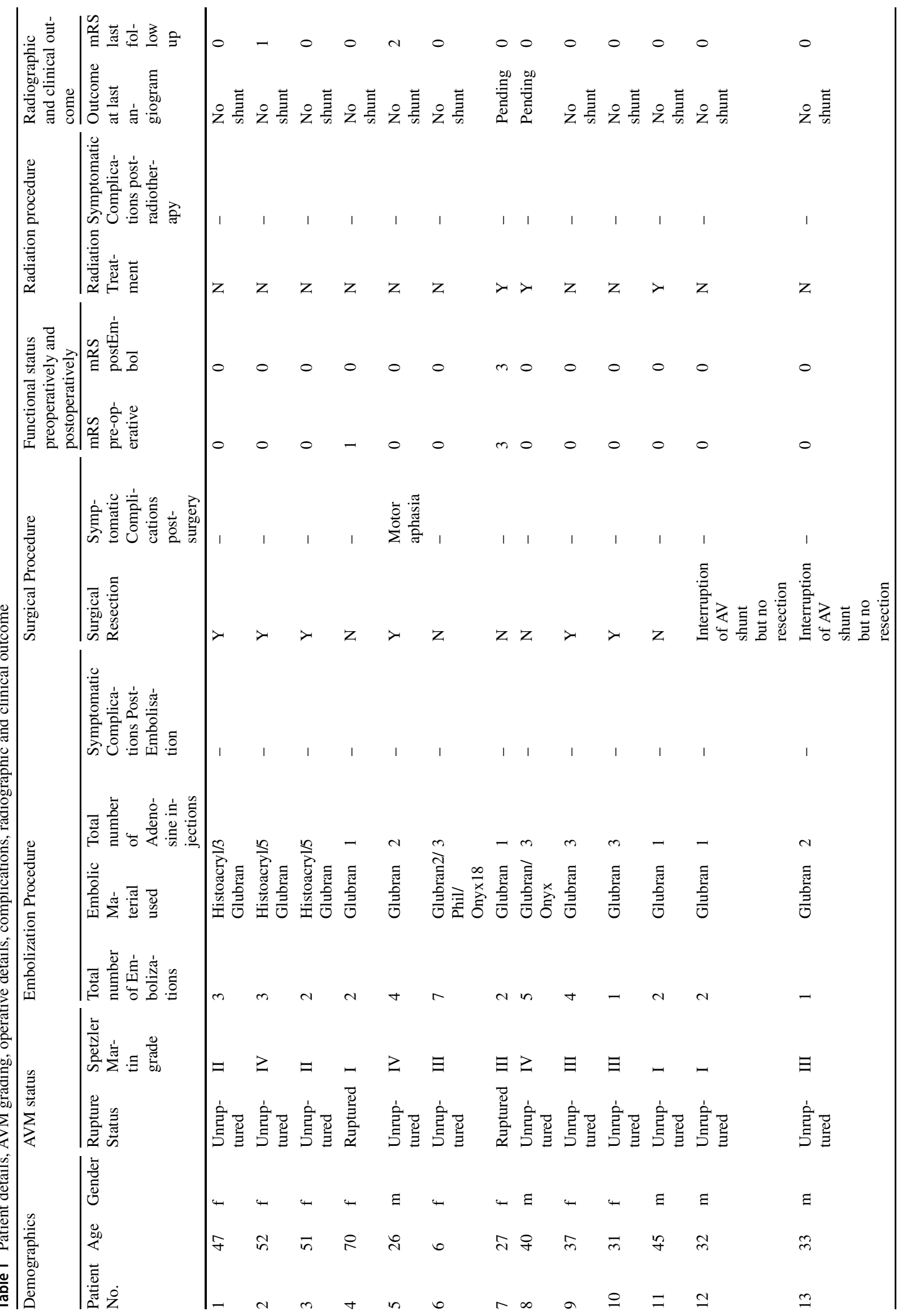




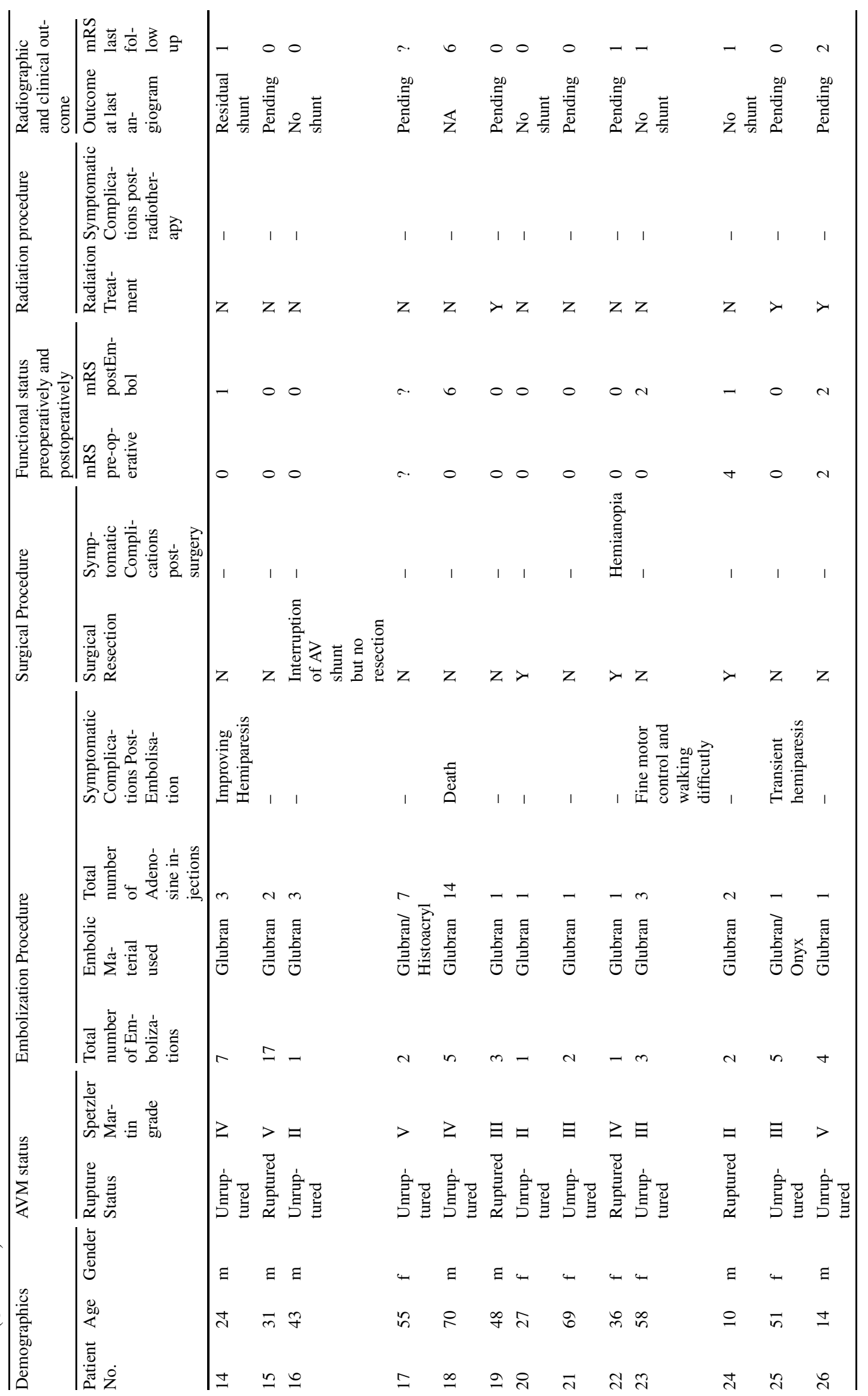




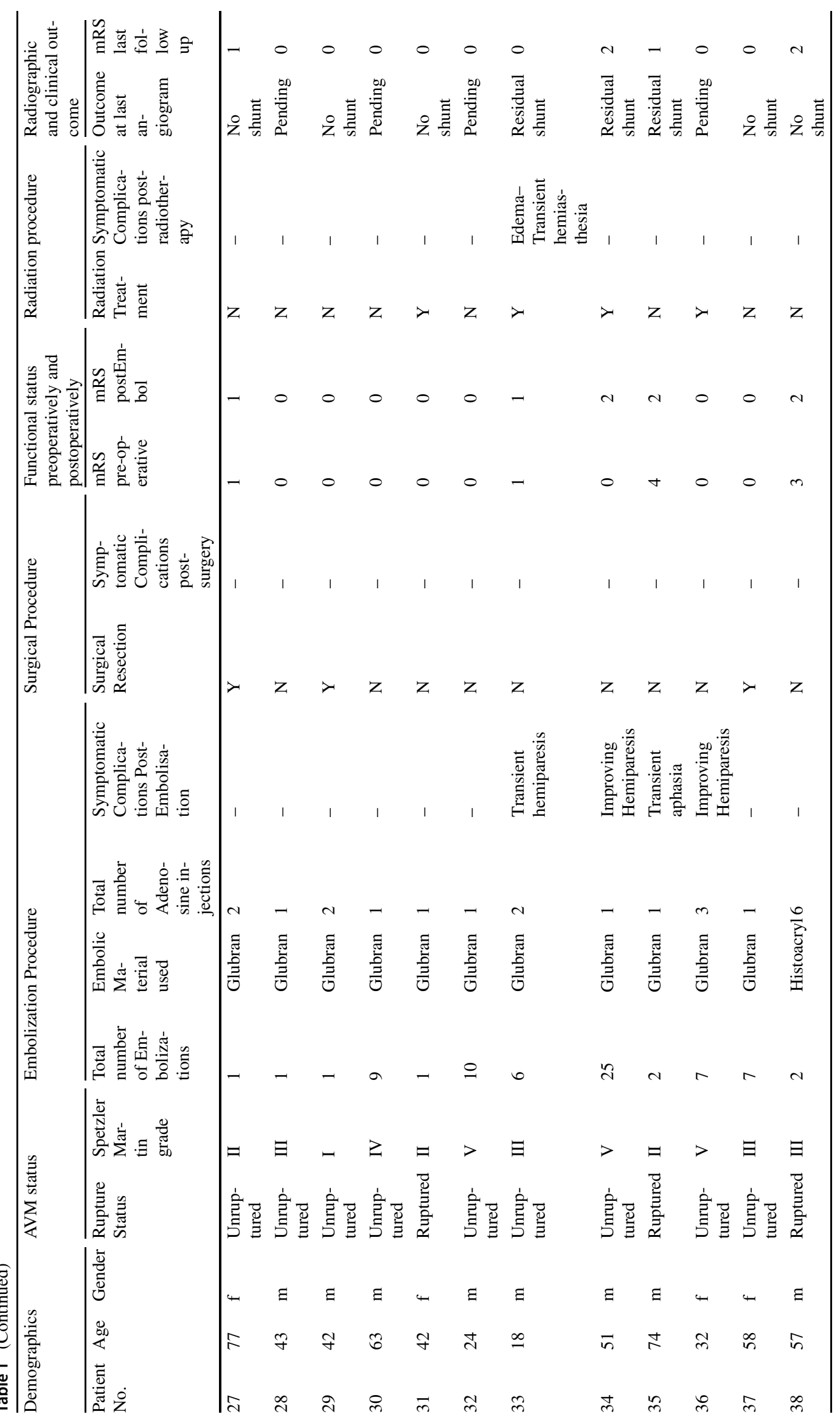




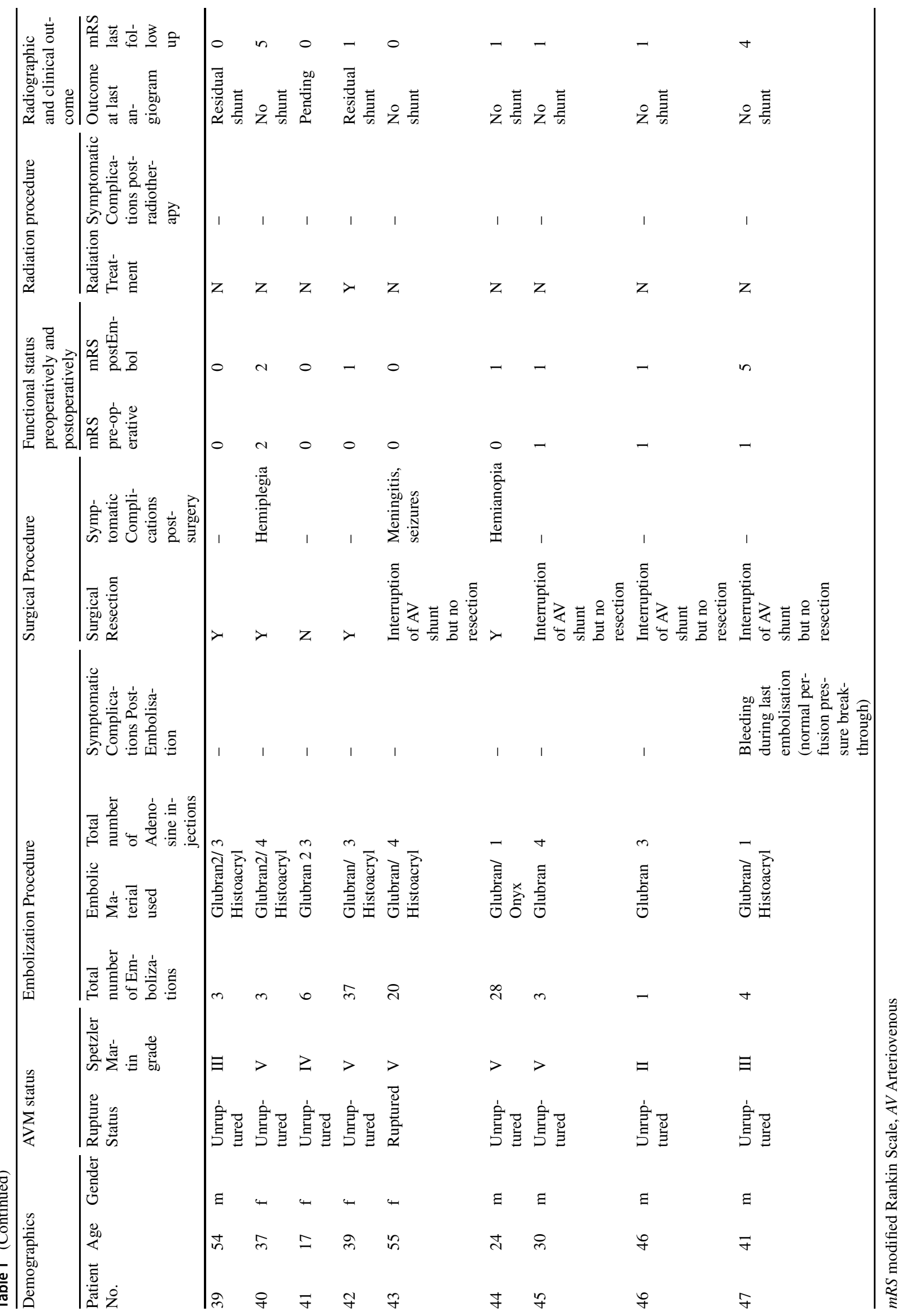


der high frame rate (e.g., 30/s) fluoroscopy. The speed and volume of glue injection has to be calibrated in a way that avoids both venous passage and proximal reflux. Fixation of the microcatheter by the glue cast is avoided by rapid removal of the microcatheter. A few millimeters glue reflux around the tip of the microcatheter can be accepted since the microcatheter can still be separated from the polymer without difficulty.

\section{Results}

We identified 47 patients (22 female, 47\%) with average age of $42 \pm 17$ years (range 6-77 range) who had undergone AVM embolization procedures using adjunctive circulatory standstill with adenosine (Table 1). In total there were 4 Spetzler Martin grade 1 (9\%), 9 grade 2 (18\%), 15 grade $3(32 \%), 8$ grade $4(18 \%)$, and 11 grade $5(23 \%)$ lesions. Of the AVMs six were ruptured or had previously ruptured, whereas 41 were unruptured (Figs. 1, 2 and 3).

The average number of embolization procedures per patient was $5.7 \pm 7.6$ (range 1-37) with an average of 2.6 2.2 (range 1-14) embolization procedures performed with the use of adenosine. In the vast majority of cases Glubran or Glubran 2 was used but in 13 cases 2 different embolic agents were used.
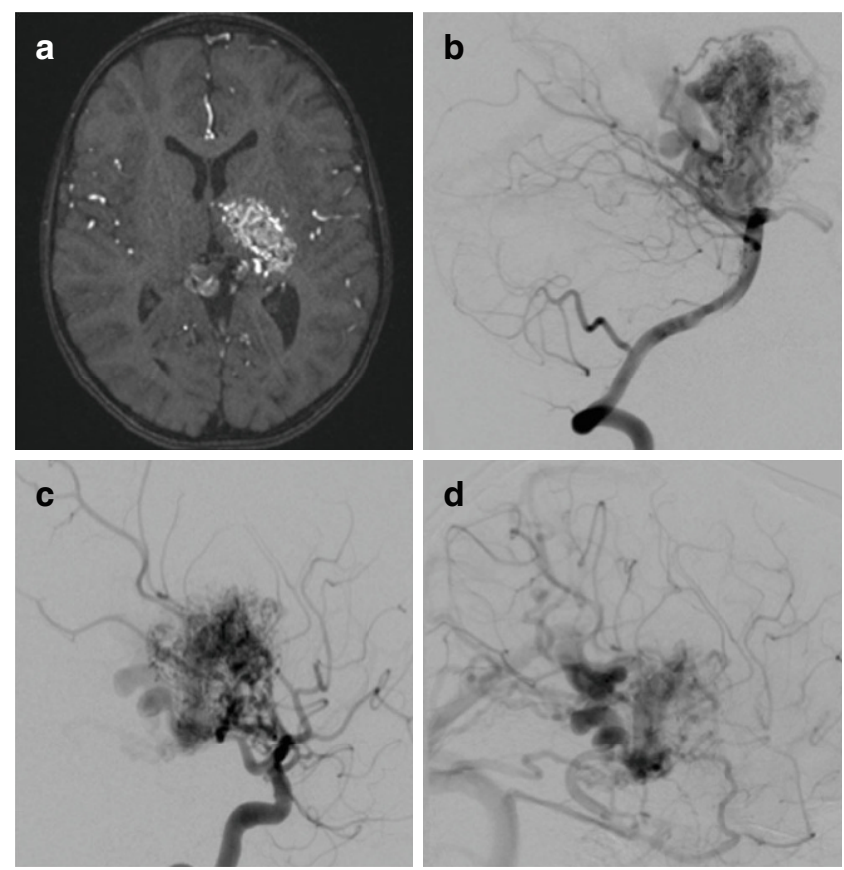

Fig. 1 A patient with a left thalamic and ganglion AVM as demonstrated on the axial TOF MRA (a). Supply to the AVM was derived from the posterior circulation (b) and the anterior circulation (c) after contrast injection into the vertebral and internal carotid arteries respectively. On the delayed angiographic images, predominantly deep venous drainage was seen $(\mathbf{d})$
Overall morbidity was $17 \%(n=8 / 47)$ and mortality $2.1 \%(n=1 / 47)$, with permanent morbidity seen in $10.6 \%$ ( $n=5 / 47)$ post-embolization. Of these complications three were transient hemiparesis and in a further three cases there was an improving hemiparesis. In one case hemorrhage post-embolization occurred. In total 23 patients underwent surgery post-embolization with interruption of the residual shunt but no resection of the AVM in 7 cases and in 16 cases there was removal of the AVM. There were 5 complications postoperatively $(5 / 23,21.7 \%)$.

In total 11 patients underwent radiosurgery after embolization and in 1 patient embolization, surgery, and radiosurgery were performed. Of the 11 patients who underwent radiosurgery there was a single complication (edema and transient hemianesthesia 9.1\%).

Angiographic follow-up was available for 32 patients with no residual shunt seen in $26(81 \%)$ and residual shunts seen in 6 patients $(19 \%)$. The angiographic follow-up is still pending in 14 patients.

At baseline, 43 patients were recorded as $\mathrm{mRS} \leq 2$ (93.5\%, $n=46)$ with clinical follow-up available in 46 patients of whom 43 patients were recorded as $\mathrm{mRS} \leq 2$ $(93.5 \%)$.

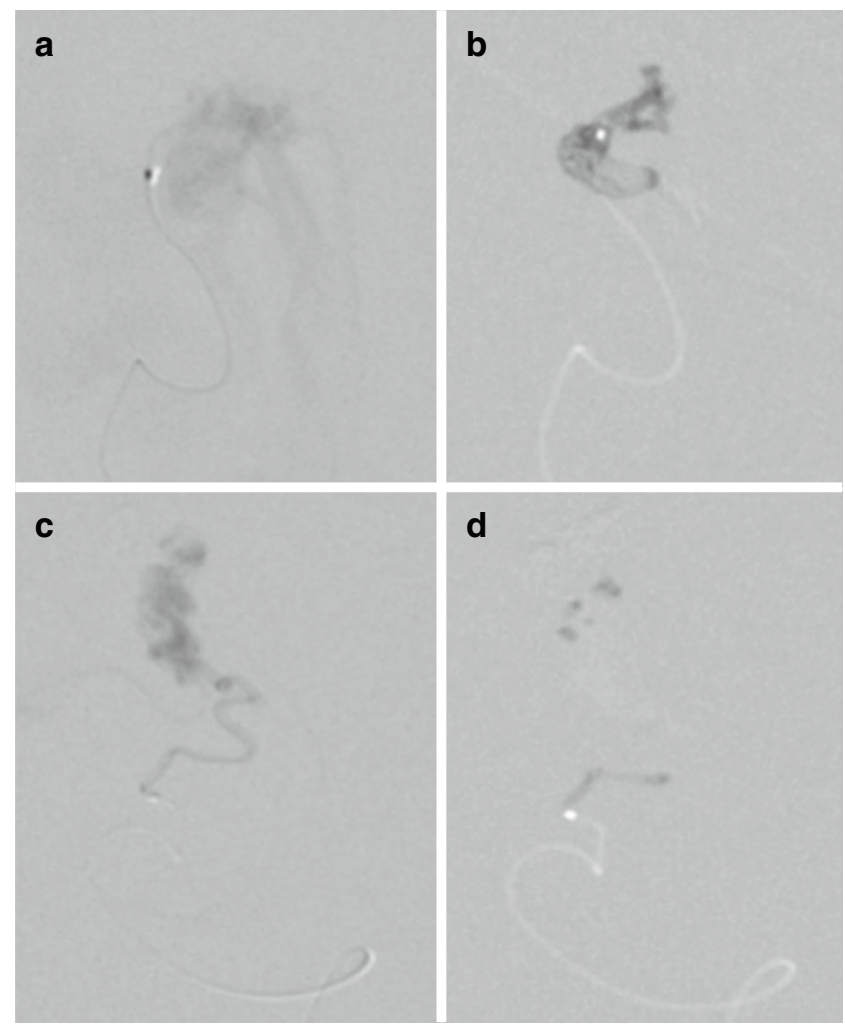

Fig. 2 Multiple embolizations were undertaken over a period of several months. Microcatheter angiography via the anterior choroidal artery (a) demonstrated rapid shunting. Embolization under asystole (16s) resulted in good nidal penetration and glue cast formation (b). A further embolization via the posterolateral choroidal branch $(\mathbf{c}, \mathbf{d})$ but without induced asystole resulted in poor nidal penetration 
Fig. 3 The angiographic appearances pre-embolization (a) and post-multiple embolization sessions including those performed under asystole demonstrated a significant reduction in the volume of the nidus (b). An axial T2-weighted magnetic resonance imaging (MRI) postembolization demonstrated continued shunting but no major infarction (c). Gamma knife radiosurgery was performed to complete the treatment, which caused some perinidal edema. (d) At year 4 post-radiosurgery there was complete obliteration of the AVM (e)
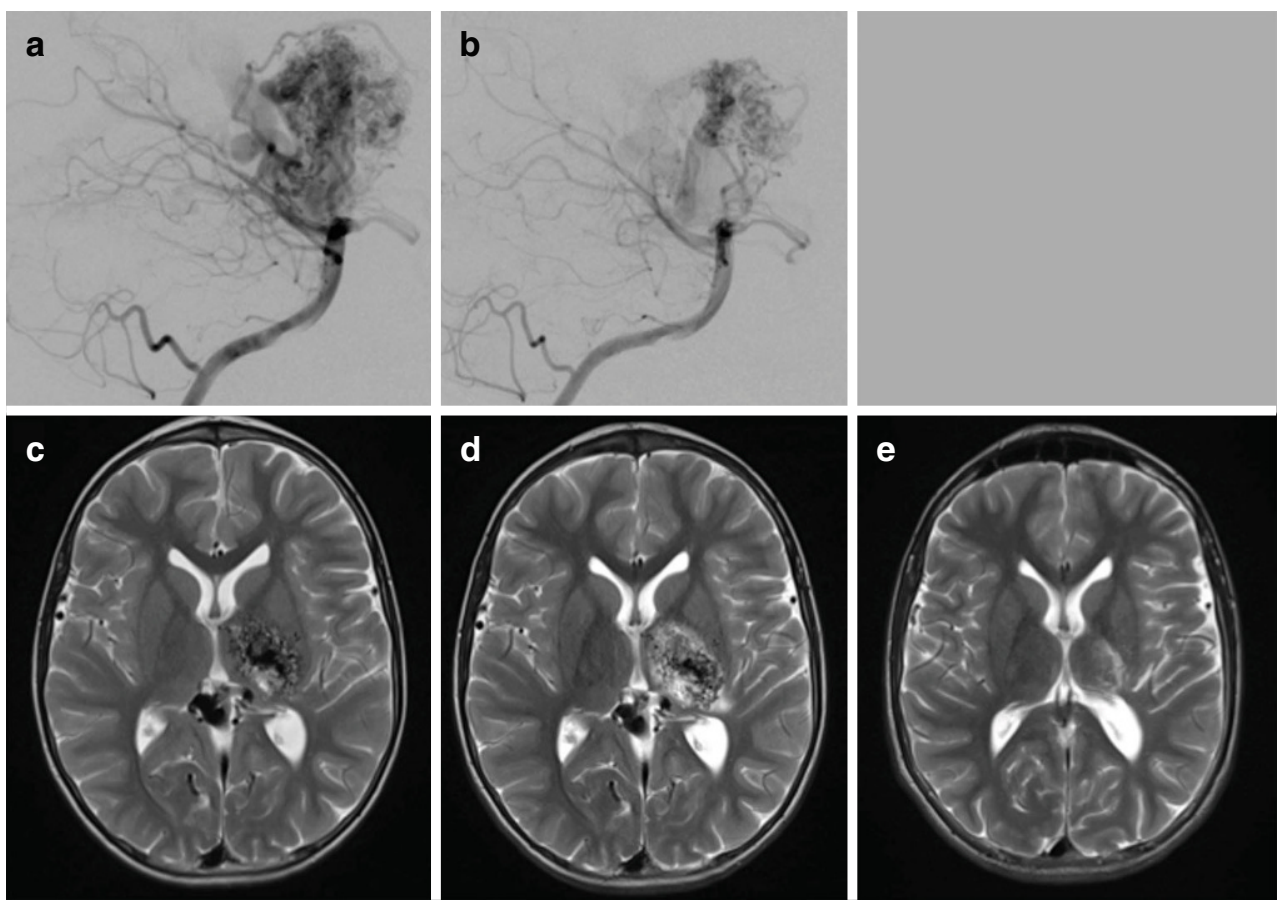

\section{Discussion}

This article presents the largest series to date on the use of adenosine induced cardiac standstill in the embolization of high flow AVMs. The results suggest that the embolization of AVMs under cardiac standstill with adenosine carries an overall risk of morbimortality close to $20 \%$; however, the risk of death is low and the majority of complications do not result in permanent disability. Virtually all patients in our cohort were $\mathrm{mRS} \leq 2$ at last follow-up.

Pile-Spellman et al. [1] were the first to report the use of adenosine induced cardiac stand still in 1999. In their case, a 40-year-old woman presented with a small intracranial hemorrhage and new onset tonic-clonic seizures and was found to have a $4 \times 5 \mathrm{~cm}$ AVM in the medial anterior occipital lobe on MRI. Angiography revealed a very highflow AVM with arterial supply principally via the posterior cerebral artery with some collaterals from the middle cerebral artery as well as transdural supply from the middle meningeal vessels. At the initial embolization the transdural supply was occluded. At the second session they felt that systemic hypotension to a mean arterial pressure (MAP) of $40-50 \mathrm{~mm} \mathrm{Hg}$ would not be sufficient to prevent venous passage of the embolic agent given the extremely highflow nature of the AVM. Therefore, it was decided to induce a cardiac pause of approximately 10-15 s during which the NBCA could be safely injected. Once in position with a Magic $1.8 \mathrm{~F}$ microcatheter (Balt Extrusion, Montmorency, France) the authors initially tried an IV bolus dose of $12 \mathrm{mg}$ of adenosine via the femoral sheath; however, this resulted in atrioventricular blockade (AVB) of only 2 cardiac cy- cles. Subsequently, dosages of $30 \mathrm{mg}$ and $48 \mathrm{mg}$ were tried; however, neither resulted in a cardiac pause of sufficient length and a dose of $64 \mathrm{mg}$ was needed to induce asystole for between 10-15 s. During the test doses contrast medium was injected through the microcatheter to ensure there was no flow reversal in the feeding artery of the AVM during the asystolic period. The authors reported a drop in the systemic MAP from $80 \mathrm{~mm} \mathrm{Hg}$ to $20 \mathrm{~mm} \mathrm{Hg}$ and during the cardiac pause nBCA (1:1 mixture of nBCA with Lipiodol) was injected resulting in obliteration of the AVM. There were no neurological or cardiovascular complications and the patient was discharged 2 days postprocedure with a plan for further embolization prior to definitive surgical resection. The authors also noted that there was a rebound in the systemic MAP after the asystolic period and that this could theoretically cause migration of glue into the draining vein and hence rupture of the AVM depending on the angioarchitecture. For this reason, they recommended mild systemic vasodilation and with nitroprusside coupled with a gradual return to baseline pressures and in this case the authors premedicated the patient with $30 \mathrm{mg}$ of nimodipine. The authors noted that one advantage of this technique over the use of systemic agents, such as esmolol, to induce hypotension is the prolonged effect on the cardiovascular system that these agents can have. In contrast adenosine has a half-life of approximately $10 \mathrm{~s}$ and in the event of a failure to revert to sinus rhythm in a timely fashion external ventricular pacing can be instigated until the adenosine induced AV blockade terminates. A rebound increase of the arterial blood pressure after the adenosine induced asystole may occur. The uncontrolled distal migration of the 
polymerized glue is unlikely since nBCA is different from ethylene-vinyl alcohol copolymer (Onyx, Medtronic) in becoming firmly attached to the vessel wall during polymerization. Distal migration of incompletely polymerized glue and hemorrhagic complications induced by undue arterial hypertension remain a concern. It is therefore recommended to avoid any increase in the systolic arterial blood pressure over $120 \mathrm{~mm} \mathrm{Hg}$. The intravenous injection of $0.5 \mathrm{mg}$ glyceryl trinitrate has been sufficient to counteract rebound arterial hypertension instantaneously.

A subsequent study by several members of the same group investigated the dose response to adenosine in 5 patients, 4 adults and 1 child, undergoing AVM embolization with adenosine induced asystole. In total 29 injections of adenosine were performed across 7 procedures. Between two and five injections of adenosine were performed to estimate the optimal dose required with the initial test bolus being between $0.25-0.35 \mathrm{mg} / \mathrm{kg}$. Subsequent doses were escalated by $10-20 \mathrm{mg}$ after an interval of 3-10 min until a 20-30 s stable MAP reduction to $25-30 \mathrm{~mm} \mathrm{Hg}$. To counteract the rebound post-adenosine hypertension a sodium nitroprusside infusion $(\approx 1 \mu \mathrm{g} / \mathrm{min} / \mathrm{kg})$ was titrated to lower the baseline MAP by about $10 \%$. The mean adenosine dose was $0.98 \pm 0.4 \mathrm{mg} / \mathrm{kg}$ with a range between $0.24-1.76 \mathrm{mg} / \mathrm{kg}$ (6-90 mg) across the participants. The duration of asystole, defined as longest R-R interval observed after the adenosine injection, was $8 \pm 3 \mathrm{~s}$. The duration of MAP $<30 \mathrm{~mm} \mathrm{Hg}$ and MAP $<50 \mathrm{~mm} \mathrm{Hg}$ was $18 \pm 12 \mathrm{~s}$ and $50 \pm 29$ s, respectively. The authors noted a linear relationship between the dose and the duration of asystole, MAP $<30 \mathrm{~mm} \mathrm{Hg}$ and $<50 \mathrm{~mm} \mathrm{Hg}$. In our experience we use a fixed dose of $36 \mathrm{mg}$ of adenosine injected directly into a venous catheter the tip being placed in the right atrium, which induces asystole of 10-20 s in the majority of patients and as such we do not typically alter the dose or carry out test bolus injections. One advantage of AVM embolization with cardiac standstill is the global effect on flow within the AVM. The use of microballoons to decrease flow from a particular artery may have unpredictable consequences on the flow within the remainder of the AVM that receives a different arterial supply. This is not the case with cardiac standstill since the flow across all arterial inputs is reduced.

Rapid ventricular pacing (RVP) is a potential alternative to adenosine induced asystole and became popular in the context of transcatheter aortic valve replacement (TAVR) [16]. We have limited experience with RVP since the logistics in the setting of AVM embolization are more complex than for adenosine induced asystole. The main advantage of RVP is the possible longer duration (up to $30 \mathrm{~s}$ ) of reduced cardiac output. The contraindications for adenosine (e.g., chronic obstructive pulmonary disease) do not apply. The circulatory effect, however, is less distinct. The RVP is typically producing a systolic blood pressure $\leq 50 \mathrm{~mm} \mathrm{Hg}$, but no standstill of the bloodstream. The glue injection during adenosine induced asystole on the contrary shows no flow-dependent propagation of the column of polymerizing embolic agent, which allows avoiding venous passage and occlusion.

Our study has several limitations including those inherent to a retrospective study. Although the number of patients in this series represents the largest case series to date using this method the overall numbers are still small. Similarly, several patients are awaiting delayed angiography to determine if a residual shunt is present.

\section{Conclusion}

Adenosine induced cardiac standstill represents a viable treatment strategy in high flow AVMs or AV shunts that carries a low risk of mortality and permanent neurological deficit.

Author Contribution VH-data collection, manuscript preparation, review, editing. $\mathrm{PB}-$ data collection, manuscript preparation, review, editing. MAP - data collection, manuscript preparation, review, editing. MA—data collection, manuscript preparation, review, editing. AK-data collection, manuscript preparation, review, editing. EH-data collection, manuscript preparation, review, editing. $\mathrm{OG}$-review, editing. $\mathrm{HH}$-data collection, review, guarantor.

\section{Declarations}

Conflict of interest P. Bhogal: consultant for phenox, MIVI neurosciences, Balt, serves on advisory board for Cerenovus. M. Aguilar Pérez: proctor and consultant for phenox. H. Henkes: co-founder of phenox. V. Hellstern, M. Alfter, A. Kemmling, E. Henkes and O. Ganslandt declare that they have no competing interests.

Ethical standards Ethical approval for this study was granted by the ethics commission of Baden-Württemberg (F-2018-080). Informed consent was obtained from all individual patients in those who were able to provide consent or from next of kin in those patients that could give consent themselves.

Open Access This article is licensed under a Creative Commons Attribution 4.0 International License, which permits use, sharing, adaptation, distribution and reproduction in any medium or format, as long as you give appropriate credit to the original author(s) and the source, provide a link to the Creative Commons licence, and indicate if changes were made. The images or other third party material in this article are included in the article's Creative Commons licence, unless indicated otherwise in a credit line to the material. If material is not included in the article's Creative Commons licence and your intended use is not permitted by statutory regulation or exceeds the permitted use, you will need to obtain permission directly from the copyright holder. To view a copy of this licence, visit http://creativecommons.org/licenses/by/4. $0 /$. 


\section{References}

1. Pile-Spellman J, Young WL, Joshi S, Duong H, Vang MC, Hartmann A, Kahn RA, Rubin DA, Prestigiacomo CJ, Ostapkovich ND. Adenosine-induced cardiac pause for endovascular embolization of cerebral arteriovenous malformations: technical case report. Neurosurgery. 1999;44:881-6; discussion 886-7.

2. Bebawy JF, Gupta DK, Bendok BR, Hemmer LB, Zeeni C, Avram MJ, Batjer HH, Koht A. Adenosine-induced flow arrest to facilitate intracranial aneurysm clip ligation: dose-response data and safety profile. Anesth Analg. 2010;110:1406-11.

3. Bebawy JF, Zeeni C, Sharma S, Kim ES, DeWood MS, Hemmer LB, Ramaiah VK, Bendok BR, Koht A, Gupta DK. Adenosine-induced flow arrest to facilitate intracranial aneurysm clip ligation does not worsen neurologic outcome. Anesth Analg. 2013;117:1205-10.

4. Bendok BR, Gupta DK, Rahme RJ, Eddleman CS, Adel JG, Sherma AK, Surdell DL, Bebawy JF, Koht A, Batjer HH. Adenosine for temporary flow arrest during intracranial aneurysm surgery: a single-center retrospective review. Neurosurgery. 2011;69:815-20; discussion 820-1.

5. Benech CA, Perez R, Faccani G, Trompeo AC, Cavallo S, Beninati S, Berardino M. Adenosine-induced cardiac arrest in complex cerebral aneurysms surgery: an Italian single-center experience. J Neurosurg Sci. 2014;58:87-94.

6. Guinn NR, McDonagh DL, Borel CO, Wright DR, Zomorodi AR, Powers CJ, Warner DS, Lam AM, Britz GW. Adenosine-induced transient asystole for intracranial aneurysm surgery: a retrospective review. J Neurosurg Anesthesiol. 2011;23:35-40.

7. Rangel-Castilla L, Russin JJ, Britz GW, Spetzler RF. Update on transient cardiac standstill in cerebrovascular surgery. Neurosurg Rev. 2015;38:595-602.

8. Heppner PA, Ellegala DB, Robertson N, Nemergut E, Jaganathan J, Mee E. Basilar tip aneurysm - adenosine induced asystole for the treatment of a basilar tip aneurysm following failure of temporary clipping. Acta Neurochir (Wien). 2007;149:517-20; discussion 520-1.

9. Vealey R, Koht A, Bendok BR. Multidose Adenosine Used to Facilitate Microsurgical Clipping of a Cerebral Aneurysm Complicated by Intraoperative Rupture: A Case Report. A A Case Rep. 2017;8:109-12.

10. Wright JM, Huang CL, Sharma R, Manjila S, Xu F, Dabb B, Bambakidis NC. Cardiac standstill and circulatory flow arrest in surgical treatment of intracranial aneurysms: a historical review. Neurosurg Focus. 2014;36:E10.

11. Al-Mousa A, Bose G, Hunt K, Toma AK. Adenosine-assisted neurovascular surgery: initial case series and review of literature. Neurosurg Rev. 2019;42:15-22.

12. Puri GD, Sen I, Bapuraj JR. Adenosine-induced cardiac standstill to facilitate endovascular embolisation of cerebral arteriovenous malformations in children. Anaesth Intensive Care. 2009;37:619-23.

13. Hashimoto T, Young WL, Aagaard BD, Joshi S, Ostapkovich ND, Pile-Spellman J. Adenosine-induced ventricular asystole to induce transient profound systemic hypotension in patients undergoing endovascular therapy. Dose-response characteristics. Anesthesiology. 2000;93:998-1001.

14. Tsimpas A, Chalouhi N, Halevy JD, Tjoumakaris S, Gonzalez LF, Monteith SJ, Dumont AS, Rosenwasser R, Jabbour P. The use of adenosine in the treatment of a high-flow vein of Galen malformation in an adult. J Clin Neurosci. 2014;21:1259-61.

15. Lylyk P, Chudyk J, Bleise C, Serna Candel C, Aguilar Pérez M, Henkes H. Endovascular occlusion of pial arteriovenous macrofistulae, using pCANvas1 and adenosine-induced asystole to control nBCA injection. Interv Neuroradiol. 2017;23:644-9.

16. Fefer P, Bogdan A, Grossman Y, Berkovitch A, Brodov Y, Kuperstein R, Segev A, Guetta V, Barbash IM. Impact of Rapid Ventricular Pacing on Outcome After Transcatheter Aortic Valve Replacement. J Am Heart Assoc. 2018;7:e009038. 